\title{
Oligodendrogliomas of the fourth ventricle: report of two cases ${ }^{1}$
}

\author{
JAMES GREENWOOD, JR., FRANK J. OTENASEK, AND FRANK S. YELIN \\ From the Methodist Hospital, Houston, Texas, and the Johns Hopkins Hospital, Baltimore, \\ Maryland, U.S.A.
}

Oligodendrogliomas comprise approximately $4 \%$ of the total of brain tumours, and of this group only $7 \%$ are found in the posterior fossa, including $2 \%$ in the fourth ventricle. Oligodendrogliomas of the fourth ventricle therefore comprise less than one case in 1,000 to 1,500 brain tumours $(0.08 \%)$. One cannot expect to encounter much more than one in a lifetime even in a large neurosurgical experience.

In 1928 Van Bogaert and Martin reported an oligodendroglioma filling the fourth ventricle. In 1950 Earnest, Kernohan, and Craig in a review of 200 oligodendrogliomas found 14 to be in the posterior fossa, four of which were in the fourth ventricle. Additional literature from 1928 to 1950 reported oligodendrogliomas of the posterior fossa (Banus and Bueno, 1929; Agostini, 1933; Garkarvi, 1936; Wycis, 1948), two of which were in the fourth ventricle, (Banus and Bueno, 1929; Garkavi, 1936). Thus, of a total of 25 posterior fossa tumours, seven were in the fourth ventricle, indicating that approximately $25 \%$ of the posterior fossa oligodendrogliomas were in the fourth ventricle. Another interesting fact noted by Krueger and Krupp (1952) in age distribution of posterior fossa oligodendrogliomas was that they usually made themselves known before the age of 21 , while supratentorial involvement usually became evident between 35 to 45 years of age. This would not necessarily indicate that the tumours began at an earlier age in the posterior fossa, but that perhaps symptoms began earlier since the room for expansion was more limited. The same may well be true of cerebellar astrocytomas.

\section{CASE REPORTS}

CASE 1 M.F., a white woman aged 27, was admitted to the Johns Hopkins Hospital, 6 February 1947, with the chief complaint of headache and blurring of vision, which had begun one year before her admission and had progressed in severity until she had continuous headache

'Read at Southern Neurosurgical Society meeting, 16 February, 1968, New Orleans, La., U.S.A. and attacks of blurring of vision, with occasional com-西 plete blindness, particularly when changing head position. Her husband had noted that recently she had had unusuats attacks of anger and had even been combative, which wasrare for her. She had been forgetful for about a monthand had noted ringing in the right ear for three months $\overrightarrow{\tilde{\omega}}$ There had been no seizures.

Neurological examination was essentially negativeexcept for evidence of mental dullness and slowness ino answering questions, although she was well oriented. Papilloedema of 3 dioptres was present bilateralty Radiographs of the skull were negative, but ventrionit lography showed evidence of internal hydrocephalus wish block present in the region of the posterior portion of third ventricle, which was not as well delineated as $\mathrm{An}^{-}$ anterior. Posterior fossa exploration on the same $\vec{a} y_{c}$ revealed normal appearing cerebellar hemispheres, bit splitting of the vermis for only a short distance disclosedo the enlarged fourth ventricle, filled with greyish tumour $\vec{r}$ apparently arising from the floor of the fourth ventrie? Biopsy was taken and the dura left open, and in addition a wide bone removal was carried out for decompression

Microscopic report This proved the tumour to be an oligodendroglioma. It was not felt that much had been accomplished in this apparently hopeless tumour of then fourth ventricle; her post-operative course, however, was uncomplicated except for a rather severe menta $\mathcal{P}$ condition, incontinence, and a tendency to sleep most of the day. She was discharged on the thirteenth post $₹$ operative day with possibly some diminution of the papilloedema. She continued to be dull and stuporous for some days, disoriented for time, but identifying people whom she knew. Within a few months she was able to resume her household activities and was lost sight of until 1963 (16 years after surgery), when she began to have generalized seizures with no alteration in her neurologicaf. examination and no signs of increased intracraniab pressure. On Dilantin (phenytoin sodium), until 10 Apriß 1967, she had no additional attacks and remainedo neurologically normal 20 years after her simple de compression. Radiographs now revealed possible calo cification in the tumour, which lay low in the middle. fossa and extended into the posterior fossa (Fig. 1). She carries on in a perfectly normal fashion as a housewife CASE 2 D.L.F., a 53-year-old housewife, was admitted tơి the Methodist Hospital, 7 September 1965. Severe 


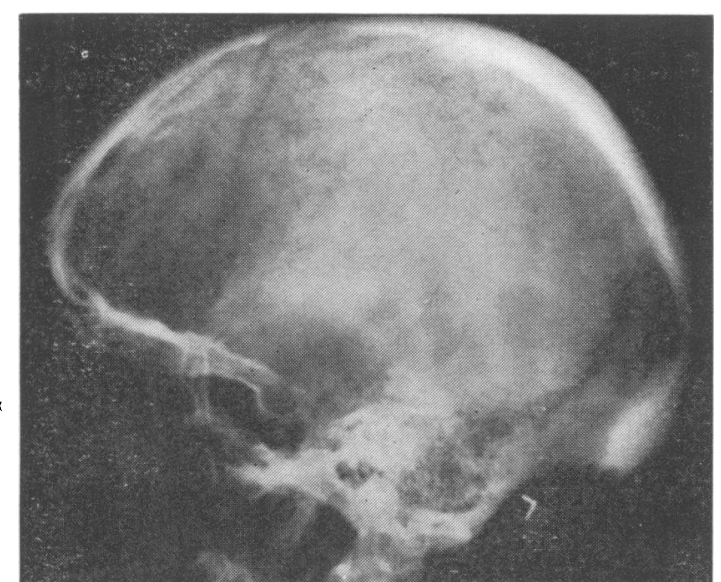

FIG. 1. Case 1 (Dr. Otenasek's patient) showing extent of suboccipital decompression. Simple biopsy was taken and patient is practically free of symptoms 20 years after surgery.

uncontrolled nausea had begun in February, several months before her hospital admission. This became progressively worse until she had had two 'blackout' attacks, the second of which resulted in a deep sleep or unconsciousness over a period of approximately 48 hours. Laboratory tests were negative, but routine skull films disclosed an abnormal calcification in the posterior fossa. Neurological examination at this time, approximately two months before her Methodist Hospital admission, showed a slight unsteadiness in gait and a tendency to deviate to either side. Co-ordination in the use of the hands was exceedingly poor and additional history indicated that there was a period of unconsciousness of approximately 24 hours in 1962 . There was some limitation of upward gaze and equivocal Babinski sign was present on the left. Careful ophthalmological examination showed very marked lack of convergence, but diplopia was reported to be greater at a distance than at close range. The left field was normal but there was some enlargement of the blind spot on the right. There was no papilloedema and at no time was there any history of significant headache.

Neurological examination showed fairly full range of extra-ocular motor function and there was no diplopia, but a definite limitation of upward gaze was noted. She exhibited a mild ataxia when walkir.g and was unable to stand or hop on either foot.

Skull films and laminograms of the posterior fossa showed a pyriform, densely calcified mass- $-3 \frac{3}{4} \mathrm{~cm}$ in length, $2 \mathrm{~cm}$ AP dimension, and $2 \frac{1}{2} \mathrm{~cm}$ maximum width -present within the mid-portion of the posterior cranial fossa; it was thought to represent a densely calcified cast of the fourth ventricle produced by a slowly growing intraventricular neoplasm, most likely an oligodendroglioma (Figs 2 and 3). There was marked erosion of the dorsum sellae.

Ventriculography on 13 October 1965 showed moder-

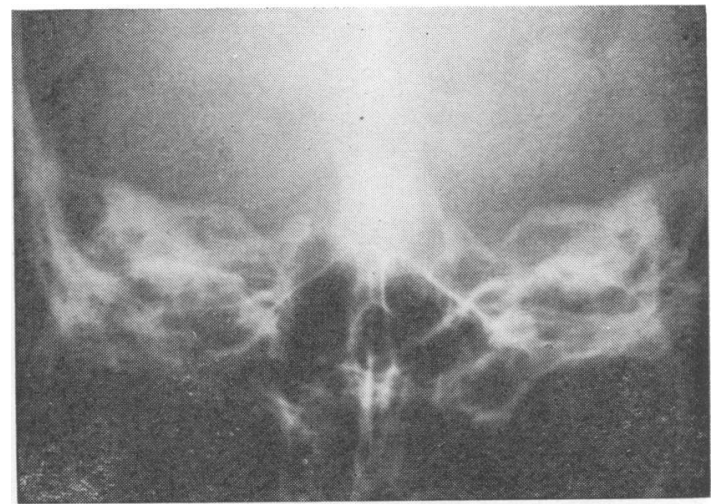

FIG. 2. AP radiograph, case 2 (Dr. Greenwood's case).

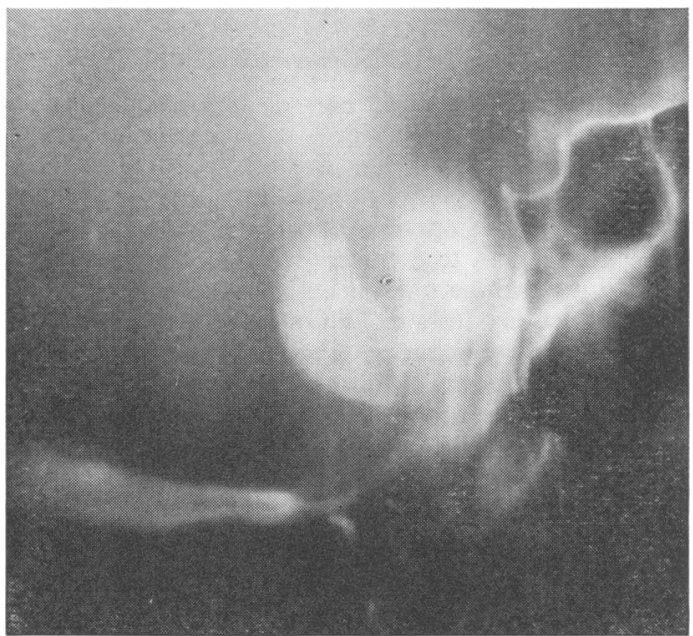

FIG. 3. Oligodendroglioma filling the fourth ventricle. Lateral laminogram (case 2).

ately severe distention of the lateral ventricles and third ventricle, secondary to obstruction of the distal aqueduct of Sylvius (Fig. 4). Suboccipital craniectomy on the same day was carried out in the upright position. Ventricular needle, placed in the right occipital burr hole, revealed that pressure was maintained on fluid introduced at the needle tip, but this was immediately reduced after removal of suboccipital bone. A midline incision in the vermis revealed a white, hard, gritty, well-circumscribed tumour, extending down to the foramen of Magendie, which was easily lifted up and was not attached to the lower portion of the walls of the fourth ventricle or its floor. Above the middle of the fourth ventricle, the tumour obviously invaded the floor of the fourth ventricle and lower aqueduct and also the lateral wall of the aqueduct on the right. Additional tumour was removed from the 


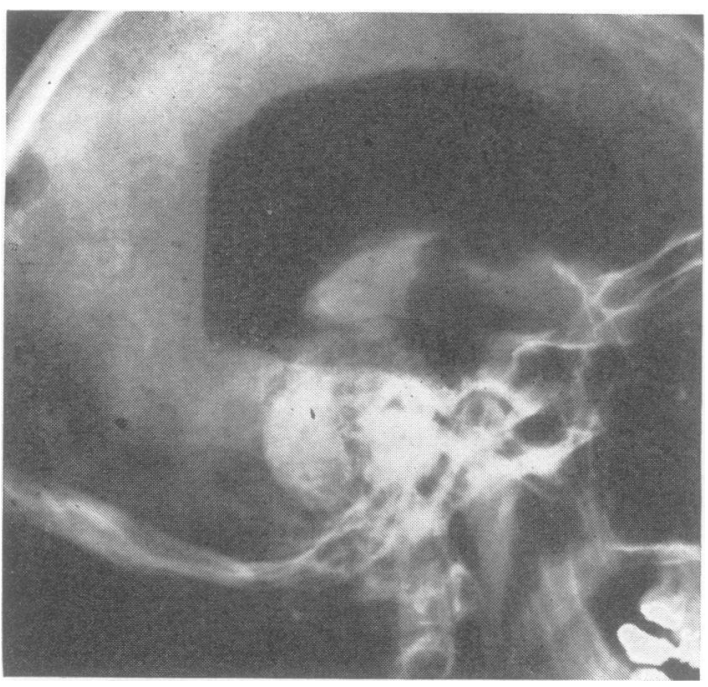

FIG. 4. Ventricululogram, case 2, showing degree of hydrocephalus.

vermis, leaving an opening into the aqueduct and third ventricle of least $\frac{1}{2}$ to $\frac{3}{4} \mathrm{~cm}$ in diameter. No attempt was made to remove the tumour infiltrating the right wall of the aqueduct, nor the small bit of tumour attached to the floor of the lower aqueduct (Fig. 4) and fourth ventricle.

Histology report 'Multiple sections of calcified material; between areas of calcification are typical nests of oligodendroglioma cells.'

Course in hospital After surgery there was difficulty in arousing her for at least six or seven days, but vital signs were essentially normal. She had a bilateral partial 3rd nerve paralysis, ptosis being most marked on the right, and evidence of skew deviation. Conjugate deviation was fairly good to the left but the right eye turned only to the right. Pupils reacted to light and she was mentally clear and entirely oriented two weeks after surgery. Her gait, however, remained rather ataxic and she was unable to walk without some support. Hand movements were excellent. Examinations of the visual fields done in April 1966, seven months after surgery, showed irregular left homonymous hemianopsia. There was difficulty in focusing the left eye; vision was difficult to evaluate but was 20/25 in the right eye. Radiographs of the skull showed practically all of the calcified tumour to have been removed (Fig. 5).

The last ophthalmological examinations revealed $20 / 25$ vision in the right eye and ability to count fingers in the left, with a partial left homonymous hemianopsia which extended across the macula in the left eye. The fundi were normal and there was no evidence of atrophy.

Her ataxia continued to be a problem but extra-ocular movements were much improved. Ptosis was slight on the left and barely discernible on the right 10 months after surgery. When last seen 13 months after surgery, she walked reasonably well without help but used a cane for support.

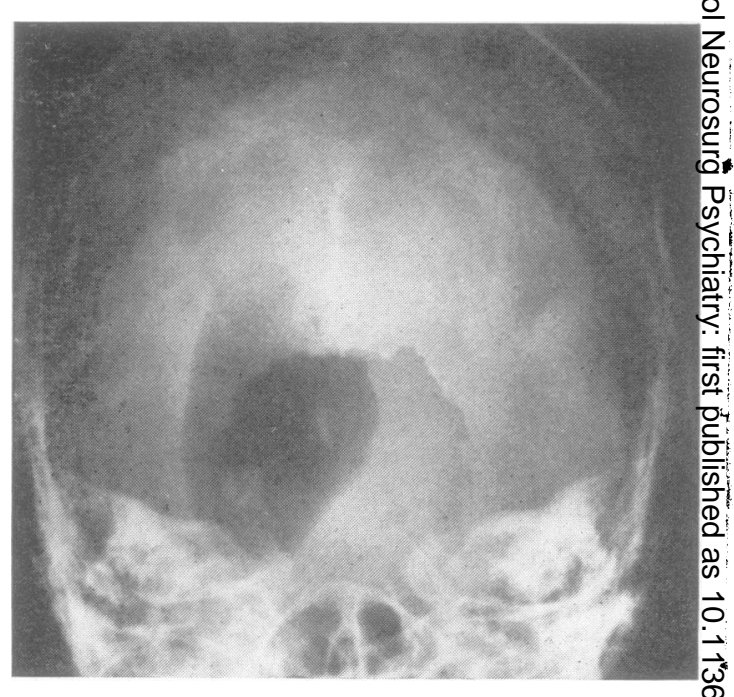

FIG. 5. Post-operative AP (case 2) showing degree of removal of calcified oligodendroglioma of the fourth ventricle.

\section{SUMMARY}

The need for recording surgical indications for ro tumours in rare locations is outlined and the ineidence of oligodendrogliomas of the fourth ventriete $0.08 \%$ in the literature, is cited (less than $1: 10805$ brain tumours). While radical brain tumour surge $\mathrm{D}$ often carries a lower mortality, less post-operat $\vec{F} e \mathrm{O}$ disability, and higher overall success, there certain instances when a more conservative approach must be considered.

Two cases of oligodendroglioma of the fourth ventricle are presented. The first case, treated byo simple decompression, is largely symptom-free $200 \mathrm{D}$ years after onset of symptoms. The second case, treated radically because of the evidence that most of the tumour lay free in the fourth ventricle, still has moderate difficulty in ambulation and vision two and a half years after removal of three-fourths to seveneights of her tumour.

\section{CONCLUSIONS}

Oligodendroglioma of the fourth ventricle is a rare,, very benign lesion, particularly when symptoms appear after 21 years of age.

Response to conservative surgery, such as simple decompression and shunt, may be excellent.

Radical, partial removal may be relatively easy, but total removal in this area is probably impossibleo and can be followed by secondary changes in 0 adjacent neurological structures which may be disabling and only slowly recovered from. 
Radical measures in certain specific brain tumours should not be used until conservative measures have been tried and proved ineffective.

\section{REFERENCES}

Agostini, C. (1933). Sopra un caso di olligodendroglioma dell, encefala. Contributo clinico e anatomo-patologico. Ann. Osp. psichiat. Perugia, 27, 1-12.

Banus, J. S., and Bueno, R. (1929). Un caso de tumor de IV ventriculo. Rev. oto-neuro-oftal. (B. Aires), 4, 515-517.
Garkarvi, C. I. (1936). Oligodendrogliomas of the cerebellum. Neuropathologiya, Psikiaria, Psikhogigiena, 5, 1775.

Earnest, F., Kernohan, J. W., and Craig, W. McK. (1950). Oligodendrogliomas: a review of two hundred cases. Arch. Neurol. Psychiat. (Chic.), 63, 964-976.

Krueger, E. G., and Krupp, G. (1952). Oligodendrogliomas arising from structures of the posterior fossa. Neurology (Minneap.), 2, 461-470.

Van Bogaert, L., and Martin, P. (1928). Les tumeurs de quatrieme ventricule et le syndrome cérébellaux de la ligne médiane: études clinques, histopathologiques et chirugicales. Rev. Neurol., 2, 431-483.

Wycis, H. T. (1948). Oligodendroglioma of the cerebellum. Arch. Neurol. Psychiat. (Chic.), 59, 404-407. 